\title{
Carotid stump syndrome: A case report
}

\author{
XU ZHANG ${ }^{1}$, SHIXIU SHAO ${ }^{2}$, XUEPING ZHENG $^{1}$, XIANG GAO ${ }^{1}$ and YONG ZHANG ${ }^{1}$ \\ Departments of ${ }^{1}$ Neurology and ${ }^{2}$ Urology, The Affiliated Hospital of Qingdao University, \\ Qingdao, Shandong 266000, P.R. China
}

Received October 17, 2014; Accepted June 22, 2015

DOI: 10.3892/etm.2015.2648

\begin{abstract}
Carotid stump syndrome (CSS) is known to be one of the causes of recurrent ipsilateral ischemic stroke following the occlusion of the internal carotid artery (ICA). The present study describes a case of left CSS in a 50-year-old patient presenting with a central retinal artery embolism following internal carotid and middle cerebral artery occlusion. The central retinal artery embolism was believed to be a consequence of microemboli, which originated from the stump of the occluded ICA, passing into the ophthalmic artery due to external carotid-internal carotid anastomotic channels, although the other possible pathophysiological causes of this condition are discussed. Digital subtraction angiography of the patient showed trickle flow in the occluded ICA during the venous phase, by which the stump emboli may have been transported to the ophthalmic artery. The patient was successfully treated with anticoagulation therapy without surgical or endovascular treatment.
\end{abstract}

\section{Introduction}

The stump of the carotid artery has been shown to be a source of emboli for ischemic stroke (1). The occurrence of ischemic stroke following carotid artery occlusion can be attributed to an embolism from the contralateral carotid artery via the circle of Willis, the distal limit of thrombus propagation, or to an embolism from the carotid stump via the external carotid artery (2). It is believed that microemboli originating from the stump of the occluded internal carotid artery (ICA) can pass into the middle cerebral artery circulation and the ophthalmic artery as a consequence of patent external carotid-internal carotid anastomosis; however, the occluded ICA is traditionally only assumed to be responsible for the symptoms once all other possible sources of emboli, such cardiac sources and atheromatous lesions in the aortic arch or ipsilateral common

Correspondence to: $\mathrm{Dr} \mathrm{Xu}$ Zhang, Department of Neurology, The Affiliated Hospital of Qingdao University, 16 Jiangsu Road, Qingdao, Shandong 266000, P.R. China

E-mail: cnxuzhang@163.com

Key words: embolism infarction, carotid stump syndrome, carotid occlusion, anticoagulation carotid artery, have been excluded. Following the exclusion of these sources, a diagnosis of carotid stump syndrome (CSS) can be made. The present study describes the case of a patient who presented with a central retinal artery embolism following ipsilateral ICA occlusion. The possible pathophysiological causes and the treatment of this case are discussed.

\section{Case report}

The present study was conducted in accordance with the Declaration of Helsinki and with approval from the Ethics Committee of Qingdao University (Qingdao, China). Written informed consent was obtained from the patient.

A 50-year-old male patient was hospitalized at 13:40 on November 21, 2013 due to a sudden weakness on the right side associated with alalia for 3 days. The cranial magnetic resonance imaging (MRI; GE Healthcare Bio-Sciences, Pittsburgh, PA, USA) revealed multiple infarctions in the left basal ganglia region and left temporal lobe. The cranial magnetic resonance angiography (MRA) indicated occlusion of the left ICA and the middle cerebral artery, and cerebral infarction was diagnosed. The symptoms did not improve significantly upon antithrombotic treatment so the patient was admitted to The Affiliated Hospital of Qingdao University (Qingdao, China).

The patient had suffered from hypertension for 17 years and smoked for $>30$ years. Physical examination upon hospitalization showed clear consciousness, incomplete motor aphasia, a 3-mm pupil diameter on both sides, reflex sensitivity to direct/indirect light, shallow right nasolabial fold, right deflection of the tongue, decreased muscular tension of the right-hand side of the body, grade 0 muscular strength and a positive Babinski sign for the right side. The findings of the cranial MRI on November 22, 2013 (Fig. 1) were consistent with the occurrence of cerebral infarction (radial-basal ganglia region, insular lobe and temporal lobe). A low-molecular-weight heparin injection (Pfizer, New York, NY, USA) was administered for anticoagulation, aspirin tablets (Bayer Schering Pharma AG, Beijing, China) were used as an antiplatelet therapy, atorvastatin calcium tablets (Pfizer) were used to regulate lipids and a hetastarch-130/0.4 NaCl injection (Beijing Fresenius Kabi Pharmaceutical Co., Ltd., Beijing, China) was used to improve infusion.

The sight of the left eye of the patient was lost at 10:10 on November 22, 2013. Physical examination showed insignificant eyelid inflammation, normal sight of the right eye, no light 
perception of the left eye, normal eye pressure, a transparent cornea, a 5-mm-diameter left pupil, no reflex to direct light and reflex sensitivity to indirect light. The diameter of the pupil of the right eye was $3 \mathrm{~mm}$. The right eye was sensitive to direct light but did not sense indirect light. In terms of the eyeground, the boundary of the optic disc of the right eye was clear and reddish, and the cup/disc ratio was $\sim 0.3$; the boundary of the optic disc of the left eye was clear and reddish, the blood vessel of the retina was thin and the retina was pale. From the ophthalmological consultation it was considered a strong possibility that occlusion of the central retinal artery of the left eye had occurred. The patient was administered oral $0.5-\mathrm{g}$ nitroglycerin tablets (Beijing Yimin Pharmaceutical Co., Ltd., Beijing, China) immediately and once a day for 2 days and was given timolol maleate (Jiangsu Chengxin Pharmaceutical Co., Ltd., Jining, China) and brimonidine D-tartrate (Allergan Pharmaceuticals Ireland Ltd., County Mayo, Ireland) eye drops. In addition, the patient was retrobulbarly injected with $10 \mathrm{mg}$ raceanisodamine hydrochloride (Suicheng Pharmaceutical Co., Ltd., Xinzheng, China) and high-pressure oxygen treatment was applied.

A cranial-cervical computed tomography (CT) angiography (GE Healthcare Bio-Sciences) was performed on November 26, 2013 (Fig. 2) and showed occlusion of the left ICA and left middle cerebral artery. Digital subtraction angiography (DSA; Philips Electronics N.V., Amsterdam, The Netherlands) was conducted on December 9, 2013 (Fig. 3) and showed a cervical occlusion of the left ICA, defective filling and vortex blood flow in the stump upon a local angiography of the left carotid artery, indicating a new thrombus in the stump. During the venous phase, a trickle blood flow was observed in the carotid artery but no flow was observed in the left middle cerebral artery. The left external carotid artery supplied blood to the brain to compensate via the deep cervical artery branch, and the image of the siphonal section of the cerebral cervical artery was weak. The anterior cerebral arteries of both sides started from the right ICA. The right ICA branch partially compensated for blood supply to the left middle cerebral artery area through the soft membrane of the anterior cerebral artery. The vertebral artery compensated partially for the left anterior circulation through the poorly developed posterior communicating artery and the soft membrane of the posterior cerebral artery, but compensation was evidently insufficient. The left ophthalmic artery showed forward blood flow.

The recurrent strokes of the patient were considered to be due to a thromboembolism originating from the left carotid artery stump. Antiplatelet agent administration was ceased and warfarin sodium tablets ( $2.5 \mathrm{mg} / \mathrm{day}$; Shanghai Xinyi Pharmaceutical Co., Ltd., Shanghai, China) were used for anticoagulation treatment. Following hospitalization for 30 days, the symptoms of the patient improved, including the incomplete motor aphasia. The patient had a defective visual field at the nasal side of the left eye, visual acuity at the level of finger count at $30 \mathrm{~cm}$ of the temporal side, a 4-mm-diameter left pupil, a dulled reflex to direct light, a sensitive reflex to indirect light and grade II muscular strength in the right side of the body. Following the patient's discharge from the hospital, atorvastatin calcium and warfarin sodium tablets were administered orally for therapy (to maintain the prothrombin time-international normalized ratio at 2.0-3.0), and rehabilitation training was performed.

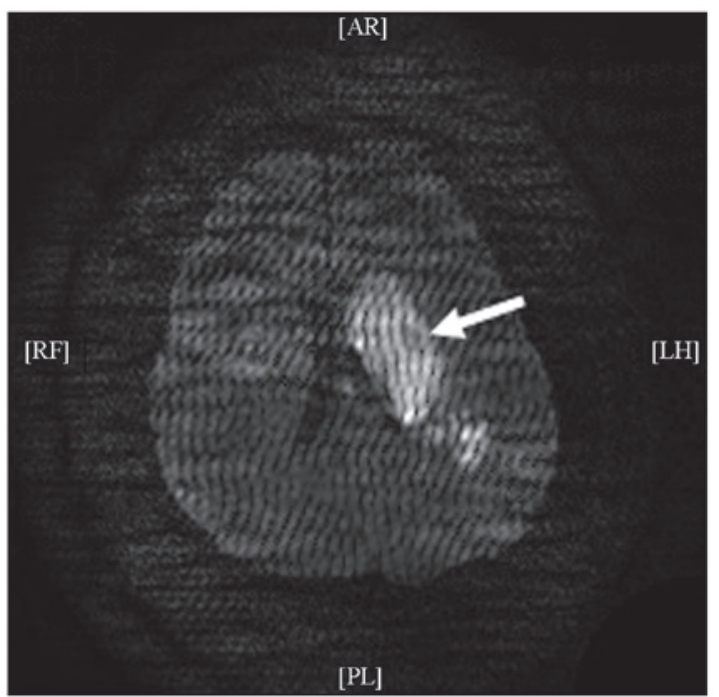

Figure 1. Diffusion-weighted image from the cranial magnetic resonance imaging of the patient showing the cerebral infarction of the left corona radiata-basal ganglia region, insular lobe and temporal lobe (arrow).

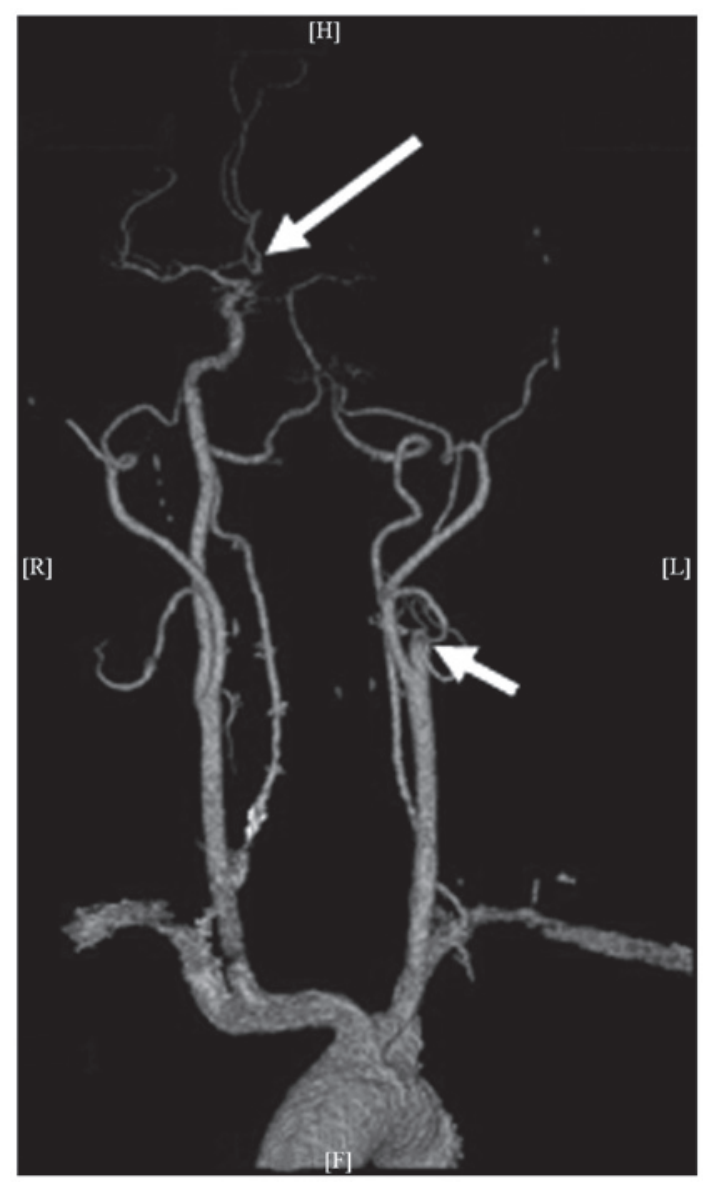

Figure 2. Cranial-cervical computed tomography angiography showing the left internal carotid artery stump (short arrow) and the right internal carotid artery that supplies blood to the ambilateral anterior cerebral artery (long arrow).

A 6-month follow-up showed that the patient's language ability, the eyesight of the left eye and the muscular strength of the right side of the body had improved significantly, and the 


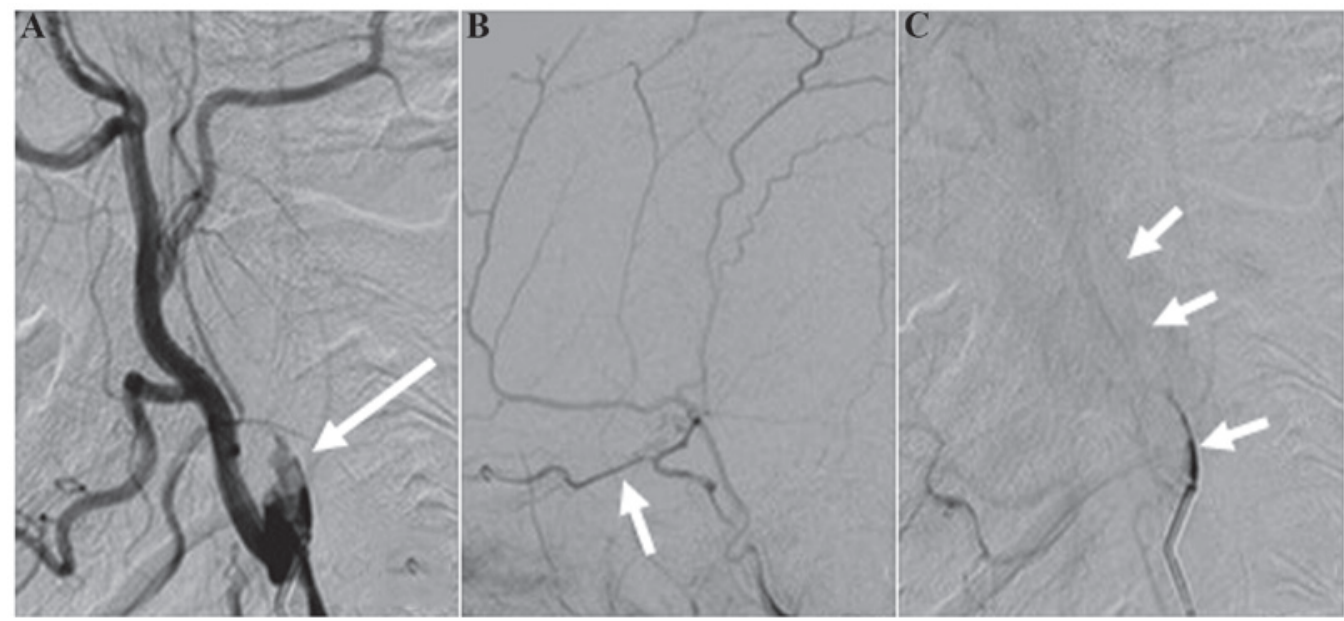

Figure 3. Cerebral digital subtraction angiography. (A) Turbulent flow in the internal carotid artery stump (arrow); (B) forward blood flow of the ophthalmic artery (arrow); (C) trickle flow of the internal carotid artery during the venous phase (arrow).

patient could walk independently. The visual acuity was finger count at $50 \mathrm{~cm}$ at the temporal side of the left eye, the diameter of the left pupil was $3 \mathrm{~mm}$, the reflex to direct light was slightly dulled and the reflex to indirect light was sensitive. In the right eye, the diameter of the pupil was $3 \mathrm{~mm}$, the reflex to direct light was sensitive and the reflex to indirect light was slightly dulled. The patient had grade IV muscular strength on the right side of the body.

\section{Discussion}

The annual incidence of mortality and stroke of patients suffering from one-sided ICA occlusion has been shown to be $\sim 30 \%$, and the risk of an ipsilateral stroke occurring in the presence of an occluded ICA is 3-5\% per year (3-5). The occurrence of ischemic stroke following carotid artery occlusion can be attributed to an embolism from the contralateral carotid artery via the circle of Willis, the distal limit of thrombus propagation, or to an embolism from the carotid bifurcation via the external carotid artery (6). When all other sources of emboli are excluded, the ipsilateral ischemic stroke resulting from an emboli arising from the stump of the occluded ICA is known as CSS. CSS was initially identified by Fields and Lemak (7). It is understood that microemboli originating from the stump of the occluded ICA or the ipsilateral external carotid artery can enter the middle cerebral artery circulation due to the existence of patent external carotid-internal carotid anastomotic channels (8).

Cranial MRI was performed on the patient at the date of the attack. Infarction was observed in the left middle cerebral artery, and the flow void signal of the left ICA and middle cerebral artery disappeared. Cranial-cerebral MRA showed left ICA and middle cerebral artery occlusion. The right-sided weakness had been attributed to the acute occlusion of the left ICA. Four days after the acute occlusion of the left ICA, the ipsilateral eyesight was lost. Once ophthalmological diseases were excluded, the loss of eyesight was suggested to be a result of the occlusion of the retinal central artery. Since the left carotid artery had been occluded, it was unclear where the embolism was from. Furthermore, no evident abnormality was observed on the patient's cardiac ultrasound and dynamic cardiogram and no clear stenosis or plaque were found on the CT angiography of the aortic arch, ipsilateral common carotid artery, external carotid artery and contra-lateral ICA. With regard to the possible mechanisms underlying the recurrent ipsilateral ischemic stroke of the patient, heart-, aortic arch-, ipsilateral common carotid artery- and external carotid artery-born emboli and low fusion could, in principle, be excluded. Cerebral angiography showed the cervical occlusion of the left ICA, deficient filling and vortex blood flow in the left carotid artery stump, and trickle flow in the ICA during the venous phase. It was presumed that the embolism was from the thrombus of the carotid artery stump. Cerebral angiography of the patient showed that the anterior cerebral arteries of both sides started from the right ICA. The terminal branch of the left ICA extends and becomes an isolated middle cerebral artery, and the middle cerebral artery had been occluded due to acute thrombus formation, which led to aphasia and right hemiplegia; therefore, the emboli derived from the ICA stump led only to ophthalmic artery ischemia.

Among the possible mechanisms of CSS, a central retinal artery embolism caused by the emboli of the ICA stump via the external carotid and ophthalmic arteries held the highest possibility. The classical pathway involves the reversed blood flow of the ophthalmic artery (ICA stump-external carotid artery-ophthalmic artery-central retinal artery). DSA of the patient showed vortex flow at the left ICA stump and a fresh thrombus, but no reversed blood flow was observed at the ipsilateral ophthalmic artery. The embolism of this patient was therefore not caused by the classical pathway mentioned above.

DSA showed that the branch of the ipsilateral deep carotid artery and the other external carotid artery opened and supplied blood to the ophthalmic artery through the siphonal section of the ICA. Emboli at the internal carotid stump could, therefore, cause the embolism of the central retinal artery and subsequent eyesight loss of the patient via the ipsilaterally open deep carotid and ophthalmic arteries (internal carotid stump-external carotid artery-deep carotid artery-siphonal section of ICA-ophthalmic artery-central retinal artery). In addition to this possible mechanism, Lakshminarayan et al (9) mentioned a mechanism of trickle flow, which indicated that 
the persistent trickle flow in the occluded ICA was a possible mechanism of CSS. DSA of the patient in the present report showed a small trickle flow in the occluded ICA during the venous phase; there was a weak image in the siphonal section of the ICA and no image in the middle cerebral artery. Although the small trickle flow in the occluded ICA could not meet with the effective perfusion of the brain, stump emboli could be transported to the ophthalmic artery. This is a possible reason why no reversed flow was observed in the ophthalmic artery. Additionally, during the venous phase, the DSA of the patient showed a small trickle flow in the left ICA and the siphonal section. Since the middle cerebral artery comprises a terminal branch of the ICA, it is unclear whether a thromboembolism in the trunk of the middle cerebral artery could, to a certain extent, be considered to be another stump. Furthermore, it is unclear whether the unstable hemodynamics in this region dispose embolus formation at the corresponding site. Since no studies, to the best of our knowledge, have investigated this mechanism, further research is required. It was considered likely that the recurrent strokes of this patient were caused by one of the aforementioned mechanisms or perhaps by a combination of at least two mechanisms.

To date, CSS therapy includes conservative medication, surgical treatment and intravascular intervention. Surgical treatment involves a longitudinal incision in the common and external carotid arteries. The inner membrane of the external carotid artery is then peeled off and the opening of the ICA is sealed so as to stop the source of emboli from the internal carotid stump. Kumar et al (10) studied 25 cases of CSS, analyzing retrospectively the clinical traits and postoperative recovery, and reported the safety and validity of surgical treatment of CSS. In a study including 40 cases of CSS, the risk of recurrent cerebral vascular events with conservative medication treatment was compared with that with surgical treatment (11). During the 4-year follow-up, only one cerebral vascular event occurred. Since the risk of recurrent stroke was low following one-sided ICA occlusion, the study did not prove the superiority of conservative medication treatment over surgical treatment; however, the prognosis of patients with CSS treated by conservative medication or surgical treatment was good (11). Despite this, compensation for an occluded ICA by the ipsilateral external carotid artery is associated with certain risks in surgical treatment, as the clamping and blocking of the external carotid artery during surgery can lead to low infusion and an ischemic stroke event.

Vascular intervention has become a new option for patients with CSS. Naylor et al (12) reported the first case of vascular intervention for patients with CSS in 2003. Stents were placed in the common carotid and external carotid arteries to block the blood flow of the internal carotid stump and to eradicate the source of the embolism. Nano et al (13) also conducted case reports concerning the intravascular intervention treatment of CSS.

In the present case, the diagnosis of CSS following a recurrent ischemic stroke was correct. There was a high possibility of a recurrent embolism; therefore, warfarin sodium was used for anticoagulation treatment. During the 6-month follow up the eyesight and physical activity of the patient improved and further strokes did not occur.

In conclusion, the occlusion of the ICA is common but reports concerning CSS cases are not. It is, therefore, necessary to enhance the understanding of CSS. For recurrent ipsilateral stroke events that occur shortly after the occlusion of an acute ICA, the possibility of CSS should be considered. The specific mechanism of CSS should be analyzed and corresponding treatment given. Anticoagulation therapy can be effective and achieve a good prognosis.

\section{References}

1. Barnett HJ, Peerless SJ and Kaufmann JC: 'Stump' on internal carotid artery - a source for further cerebral embolic ischemia. Stroke 9: 448-456, 1978.

2. Quill DS, Colgan MP and Sumner DS: Carotid stump syndrome: A colour-coded Doppler flow study. Eur J Vasc Surg 3: 79-83, 1989.

3. Eikelboom BC and Ackerstaff RG: Preoperative prediction of cerebral ischaemia due to carotid occlusion. Eur J Vasc Surg 7 (Suppl A): 21-24, 1993.

4. Irvine CD: The significance of one occluded internal carotid artery. Eur J Vasc Endovasc Surg 16: 91-93, 1998.

5. Nicholls SC, Kohler TR, Bergelin RO, Primozich JF, Lawrence RL and Strandness DE Jr: Carotid artery occlusion: Natural history. J Vasc Surg 4: 479-485, 1986.

6. Kawano H, Inatomi Y, Hirano T, Yonehara T and Uchino M: Anticoagulation therapy for vertebral artery stump syndrome. J Neurol Sci 295: 125-127, 2010.

7. Fields WS and Lemak NA: Joint study of extracranial arterial occlusion: X. Internal carotid artery occlusion. JAMA 235: 2734-2738, 1976.

8. Kawano H, Inatomi Y, Hirano $\mathrm{T}$ and Yonehara T: Vertebral artery stump syndrome in acute ischemic stroke. J Neurol Sci 324: 74-79, 2013.

9. Lakshminarayan R, Scott PM, Robinson GJ and Ettles DF: Carotid stump syndrome: Pathophysiology and endovascular treatment options. Cardiovasc Intervent Radiol 34 (Suppl 2): S48-S52, 2011.

10. Kumar SM, Wang JC, Barry MC, Farrell L, Kelly CJ, Fitzgerald PH, Leahy A and Hayes DB: Carotid stump syndrome: Outcome from surgical management. Eur J Vasc Endovasc Surg 21: 214-219, 2001.

11. Hrbáč T, Beneš V, Širůček P, Jonszta T, Herzig R, Procházka V and Skoloudík D: Safety and efficacy of surgical treatment of carotid stump syndrome: Pilot study. Ann Vasc Surg 26: 797-801, 2012.

12. Naylor AR, Bell PR and Bolia A: Endovascular treatment of carotid stump syndrome. J Vasc Surg 38: 593-595, 2003.

13. Nano G, Dalainas I, Casana R, Malacrida G and Tealdi DG: Endovascular treatment of the carotid stump syndrome. Cardiovasc Intervent Radiol 29: 140-142, 2006. 